\title{
Rasgos hidrográficos y batimétricos del Refugio Nacional de Vida Silvestre Ostional y áreas adyacentes, Guanacaste, Costa Rica
}

\section{Hydrographic and bathymetric features of the Ostional National Wildlife Refuge and adjacent areas, Guanacaste, Costa Rica}

\author{
Carlos Luis Brenes ${ }^{1 *}$, Amaru Márquez ${ }^{1}$, Wagner Quirós ${ }^{2}$ y Rosario Benavides ${ }^{1}$
}

\begin{abstract}
RESUMEN
Entre el 6 y el 11 de febrero del 2012, se realizó un levantamiento hidrográfico y batimétrico en la región situada entre Punta Guiones y Cuajiniquil, en el Pacífico norte de Costa Rica. Las temperaturas superficiales se ubicaron entre los 25.5 y $27.5^{\circ} \mathrm{C}$, y el rango salino superficial estuvo entre 33.2 y 33.8 PSU. La termoclina se localizó a los $20 \mathrm{~m}$ en el extremo norte de la región, mientras que en el extremo sur, desde Ostional hasta Guiones, se observó ligeramente más profunda, alrededor de los $25 \mathrm{~m}$. El espesor de la capa de mezcla fue de $10 \mathrm{~m}$ en toda el área estudiada. Dos giros, uno ciclónico y otro anticiclónico, se registraron en la región frente a Ostional. Los estratos superficiales estuvieron ocupados por el Agua Superficial Tropical (AST), con salinidades inferiores a $34 \mathrm{PSU}$ y temperaturas superiores a $26^{\circ} \mathrm{C}$, y los niveles intermedios $(\sim 50 \mathrm{~m})$, por el Agua Subsuperficial Subtropical (ASS), caracterizada por un núcleo salino cercano a las 35 PSU. La batimetría de la región muestra isóbatas paralelas a la costa, con una elevación alrededor de los $20 \mathrm{~m}$ de profundidad en el extremo sur de la zona estudiada. El grado de inclinación o pendientes no sobrepasaron los $14.5^{\circ}$.
\end{abstract}

Palabras claves: Masas de agua, batimetría, Pacífico, Costa Rica, Ostional.

\begin{abstract}
Between February 6 and 11,2012, a hydrographic cruise and bathymetric sounding were conducted in the region located between Guiones and Cuajiniquil, in the Northern Pacific area of Costa Rica. The range of surface temperatures is 25.5 to $27.5^{\circ} \mathrm{C}$, and the saline surface ranges between 33.2 and 33.8 PSU. The thermocline is located at $20 \mathrm{~m}$ at the northern end of the region, while at the southern end, from Ostional up to Guiones, it was observed slightly deeper, approximately $25 \mathrm{~m}$ deep. The thickness of the mixed layer was $10 \mathrm{~m}$ in virtually all the studied area. Two gyres, one cyclonic and another anticyclonic, were observed in front of Ostional. The surface layers were occupied by the Surface Tropical Water (STW), with salinities below $34 \mathrm{PSU}$ and temperatures greater than $26^{\circ} \mathrm{C}$, and the intermediate levels $(\sim 50 \mathrm{~m})$ by the Subsurface Subtropical Water (SSW), characterized by a saline core near 35 PSU. The bathymetry of the region shows isobaths parallel to the coast, with an elevation close to $20 \mathrm{~m}$ deep at the southern end of this area. The degree of inclination did not exceed $14.5^{\circ}$.
\end{abstract}

Keywords: Water masses, bathymetry, Pacific, Costa Rica, Ostional.

1 Servicio Regional de Información Oceanográfica, Universidad Nacional (SERIO-UNA).

2 International Student Volunteers (ISV-CR). Heredia, Costa Rica. cbrenes.una@gmail.com*, tupac.marquez@gmail. com,wagner.quiros@isvolunteers.org, rbmorera@gmail.com

Recibido 30-III-2012

Aceptado 9-VIII-2012

DOI: http://dx.doi.org/10.15359/revmar.4.10

Rev. Mar. Cost. ISSN 1659-455X. Vol. 4: 145-156, Diciembre 2012. 


\section{INTRODUCCIÓN}

El Refugio Nacional de Vida Silvestre Ostional (RNVSO) constituye, junto con la playa Nancite del Parque Nacional Santa Rosa, dos de las áreas más importantes en el mundo para la anidación de la tortuga lora (Lepidochelys olivacea). En esta playa se producen "arribadas" de estas tortugas, normalmente una vez al mes y por unos tres a cinco días. También llegan a la playa a desovar, en algunas ocasiones, la tortuga baula (Dermochelys coriacea) y la tortuga verde o negra (Chelonia mydas agassizii) del Pacífico. Este Refugio ha sido considerado como uno de los sitios más importantes de anidación en arribada de la tortuga lora (L. olivacea), con registros de 335555 hembras anidantes por año. Los períodos de menor anidación ocurren entre enero y abril, y los de mayor de septiembre a noviembre (Valverde, 2007).

Desde el punto de vista geológico, la zona de estudio pertenece a formaciones del Cretácico, presentando numerosas rocas ígneas, andesíticas y basaltos (Bergoing \& Brenes, 1977). El RNVSO se localiza dentro de la zona de vida del Bosque Tropical Húmedo (Tosi, 1969). El régimen mareal es mixto con dos pleamares y dos bajamares. La hidrografía cerca de la superficie y la circulación en el Pacífico norte costarricense están fuertemente influenciadas por la interacción local océano-atmósfera (Lizano, 2007; Brenes et al. 2008). Existe una señal estacional en la mayoría de las características oceanográficas de esta zona, relacionada con la migración norte-sur de la Zona de Convergencia Intertropical (ZCIT) (Kessler, 2006). Los índices termohalinos de las masas de agua superficiales son controlados no solo por flujos locales de calor, escorrentía y balance precipitación-evaporación, sino que la circulación también es controlada por la variabilidad estacional del campo de viento (Kessler, 2006; Brenes et al. 2008).

El máximo de precipitación en esta región sigue la posición de la ZCIT, consecuentemente, el mínimo estacional se localiza en abril-mayo y el máximo en octubre-noviembre (Amador et al. 2006). La evolución estacional del campo de viento también acompaña la ZCIT, en la cual sobresalen en el ámbito regional los tres pasos de montaña en los golfos de Tehuantepec, Papagayo y Panamá (Chelton et al. 2000a; 2000b) y sus respectivos chorros o "jets" eólicos que se generan entre noviembre y marzo. Estos chorros producidos por la intensificación de los campos de presión atmosférica en el Golfo de México y el mar Caribe, y el respectivo fortalecimiento de los vientos alisios del noreste, modulan al final y principio de cada año la hidrografía de todo el Pacífico centroamericano (Kessler, 2006; Fiedler \& Talley, 2006; Brenes et al. 2008). En el caso particular de la zona de estudio, los afloramientos de Papagayo son muy importantes en la dinámica que la caracteriza. Este fenómeno de afloramiento en el Golfo de Papagayo está relacionado con la generación del Domo Térmico de Costa Rica (DTCR) (Kessler, 2006; Fiedler \& Talley, 2006).

El DTCR es un afloramiento oceánico centrado en $9^{\circ} \mathrm{N}, 89^{\circ} \mathrm{W}$ y tiene forma de elipse, cuyo eje mayor se puede extender en su fase madura en dirección latitudinal muy cerca del Pacífico norte de Costa Rica (septiembre), influenciando también en las características oceanográficas de la región antes mencionada (Fiedler \& Talley, 2006).

Varios factores ambientales, como la inclinación y composición de las playas, mareas y fases lunares, han sido asociados 
con el fenómeno de las arribadas. Sin embargo, a pesar de la importancia pesquera y ecológica de la zona de este Refugio y áreas adyacentes, no se han hecho estudios batimétricos ni se han caracterizado sus masas de agua, factores que influyen en la distribución de las especies e incluso afectan la ocurrencia del fenómeno antes citado.

La incorporación de información ambiental, que permita correlacionar en el futuro los patrones migratorios de las tortugas y sus hábitats en esta zona del Pacífico norte de Costa Rica, es un elemento importante en el manejo de estas áreas marinas.

Los objetivos del presente trabajo son caracterizar, por primera vez, la batimetría de la zona en mención, así como determinar las características termohalinas de las masas de agua presentes en dicha región, con la finalidad de que los resultados obtenidos sean útiles para estudios posteriores del comportamiento del recurso marino de la zona.

\section{METODOLOGÍA}

El trabajo de campo se llevó a cabo durante un crucero hidrográfico realizado entre el 6 y el 11 de febrero del 2012. Se realizaron cinco transectos perpendiculares a la costa con una longitud de $9 \mathrm{~km}$ cada uno, en la zona comprendida entre Punta Guiones y Punta Cóncavas (Fig. 1).

Se muestreó en 30 estaciones hidrográficas con un perfilador vertical de temperatura, conductividad y profundidad, conocido por sus siglas en inglés como CTD, de la casa Falmouth Scientific. Las estaciones estuvieron separadas de forma equidistante $1.7 \mathrm{~km}$. Los datos batimétricos se obtuvieron simultáneamente durante la navegación, con una Ecosonda Garmin 421S, y se les aplicó la corrección mareal pertinente. Los gráficos se elaboraron con SURFER 10 (2011).

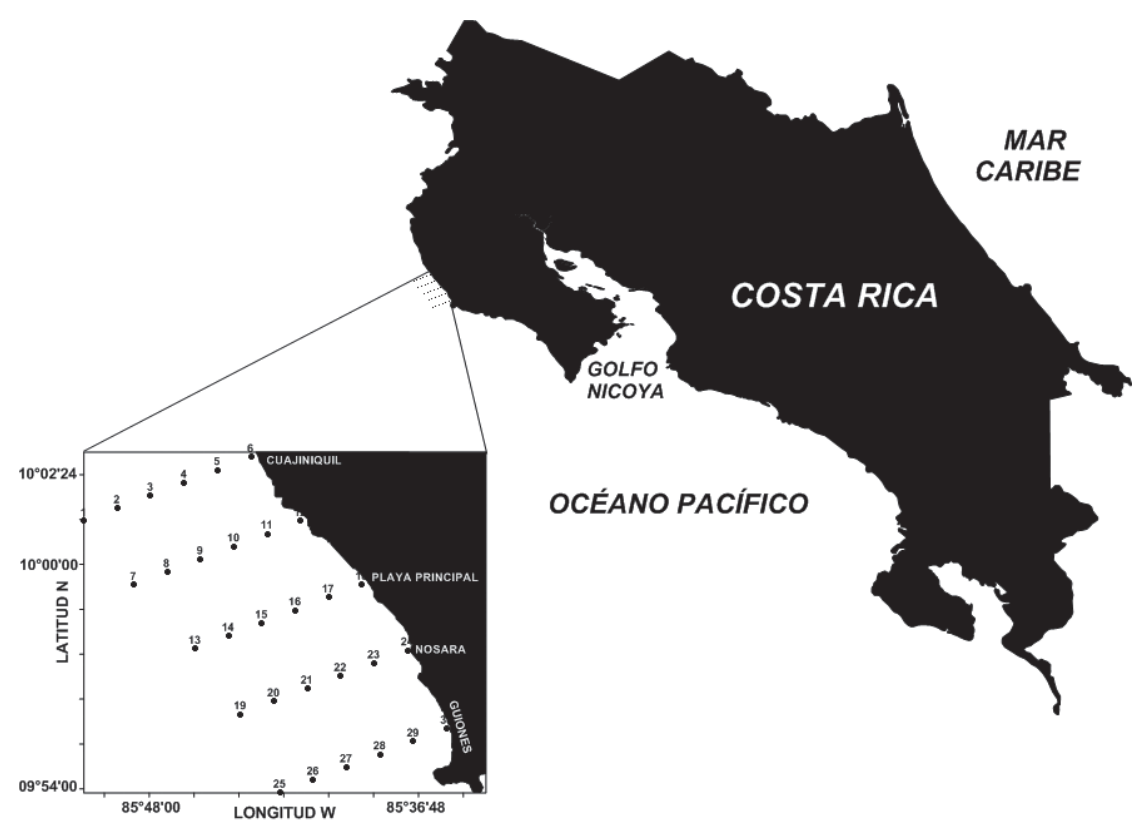

Fig. 1. Área de estudio y ubicación de estaciones hidrográficas

Fig. 1. Study area and location of hydrographic stations 
Se elaboraron imágenes de satélite para obtener los campos superficiales de temperatura y clorofila-a en las regiones adyacentes a la zona de estudio, utilizando el GES-DISC Interactive Online Visualization and Analysis Infrastructure, Goddard Earth Sciences (GES) Data and Information Services Center (DISC), NASA.

\section{RESULTADOS Y DISCUSIÓN}

Durante el período de muestreo, todo el Pacífico norte de Costa Rica presentó temperaturas superficiales relativamente bajas $\left(26<\mathrm{T}>27^{\circ} \mathrm{C}\right)$ y concentraciones de clorofila-a altas $\left(\sim 1 \mathrm{mg} / \mathrm{m}^{3}\right)$, como consecuencia del afloramiento de Papagayo (no se muestran todas las imágenes, Fig. 2). Durante los meses de enero a abril existe un aceleramiento del viento alisio en el Caribe. Este viento pasa a través de las llanuras al norte de nuestro país y se proyecta hacia el Pacífico, convirtiéndose en una corriente en chorro al frente del Golfo de Papagayo. De esta manera en el Pacífico norte de Costa Rica, el viento sigue siendo de componente noreste (Lizano, 2007).

Swimmer et al. (2009) estudiaron las posibles rutas migratorias para las tortugas lora en la región oceánica pacífica frente a

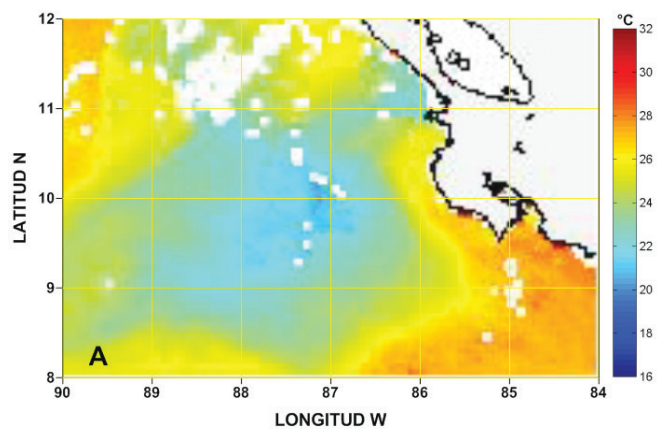

Costa Rica, concluyendo que estas tortugas permanecieron en un rango de temperatura superficial entre 23.3 y $30.5^{\circ} \mathrm{C}$ (promedio de $27.1^{\circ} \mathrm{C}$ ), y en áreas donde las concentraciones de clorofila-a fueron de $0.37 \mathrm{mg} / \mathrm{m}^{-3}$. Estudios previos (Polovina et al. 2001; 2004) sugieren que las zonas de afloramiento son los hábitats preferidos por estos organismos, donde son frecuentes los frentes oceanográficos y remolinos.

Como fue comentado previamente, estos fenómenos suelen ocurrir entre noviembre y marzo de cada año, y tienen una periodicidad cercana a los 10 días. Su importancia está en que las aguas que emergen de los niveles intermedios de la columna de agua enriquecen con sales nutritivas todo el estrato superior, beneficiando el recurso vivo de la zona.

La distribución espacial de las temperaturas superficiales se ubicó entre los 25.5 y $27.5^{\circ} \mathrm{C}$ (Fig. 3A). Dos núcleos de aguas relativamente frías se localizaron en las zonas ubicadas al sur y al norte de Ostional. Los valores de temperatura más altos se presentaron a unos $3 \mathrm{~km}$ frente a esta playa, y la forma de las isotermas en dirección a la línea de costa muestra una influencia directa de las aguas costeras adyacentes. Este mismo patrón se contempla

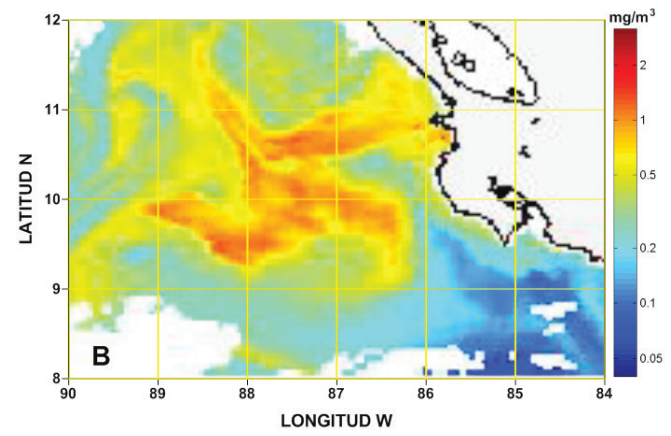

Fig. 2. A) Temperatura superficial $\left({ }^{\circ} \mathrm{C}\right)$ y B) clorofila-a superficial $\left(\mathrm{mg} / \mathrm{m}^{3}\right)$ para el día $11 \mathrm{de}$ febrero del 2012 (imágenes producidas a partir de satélites NOAA-GOES 10,12 y Aqua/MODIS)

Fig. 2. A) Surface temperature $\left({ }^{\circ} \mathrm{C}\right)$ and B) surface chlorophyll-a $\left(\mathrm{mg} / \mathrm{m}^{3}\right)$ for February 11,2012 (images produced from NOAA-GOES 10.12 and Aqua / MODIS satellites) 
a $10 \mathrm{~m}$ de profundidad, donde los mayores valores de temperatura se observan muy cerca de la zona anterior. A partir de los 20 $\mathrm{m}$, las aguas más cálidas se desplazan al norte de San Juanillo. La cuantificación de estos campos térmicos es importante, pues estudios anteriores (Cornelius \& Robinson, 1987; Swimmer et al. 2006; Plotkin, 2010) sugieren que existe una correlación entre variables oceanográficas, como la temperatura superficial y la concentración de clorofila-a, con el movimiento de las tortugas, y que variaciones drásticas en los campos de temperatura podrían tener una influencia directa sobre los patrones migratorios de estos organismos y en la disminución en las arribadas de tortugas a la zona. Un ejemplo de este efecto sucede durante la fase cálida del ENOS (El Niño-Oscilación del Sur), en

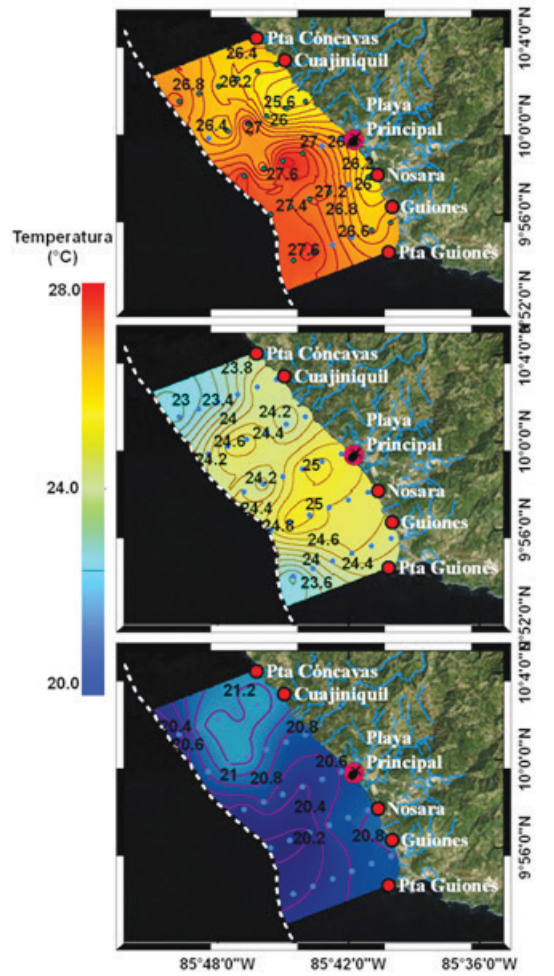

la cual cambios climáticos temporales, que afectan inicialmente la abundancia de organismos planctónicos, repercuten en el ciclo biológico de todos los demás organismos del ecosistema, observándose un retraso en su arribo a las zonas de reproducción y anidación y la temporada se acorta, disminuyendo también el total de nidos producidos por cada especie (Polovina et al. 2001). La zona del RNVSO está cíclicamente sometida a la influencia de El Niño, de manera que los resultados del presente trabajo son importantes para evaluar variaciones futuras asociadas a dicho fenómeno.

La salinidad mostró un comportamiento espacial poco homogéneo en la superficie y hasta los $10 \mathrm{~m}$ de profundidad (Fig. 3D, 3E). El aporte de agua dulce por escorrentía es uno de los factores que más

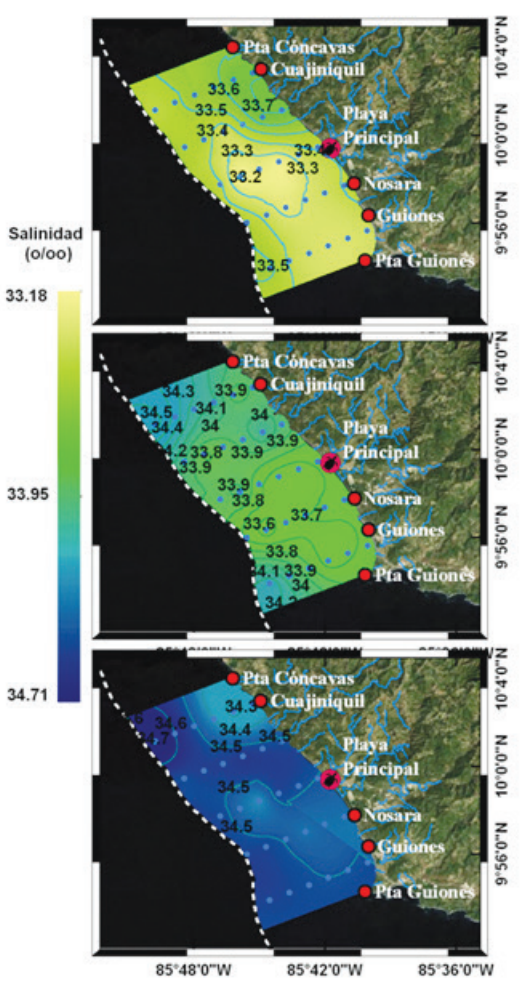

Fig. 3. Distribución espacial de la temperatura $\left({ }^{\circ} \mathrm{C}\right)$ y salinidad (PSU): superficial (A) y (D), $10 \mathrm{~m}$ (B) y (E), $20 \mathrm{~m}(\mathrm{C})$ y (F)

Fig. 3. Spatial distribution of temperature $\left({ }^{\circ} \mathrm{C}\right)$ and salinity (PSU): surface (A) and (D), $10 \mathrm{~m}$ (B) and (E), $20 \mathrm{~m}(\mathrm{C})$ and $(\mathrm{F})$ 
modulan el campo superficial salino en aguas costeras. Los caudales que drenan hacia el Pacífico atravesando el RNVSO son: Quebrada el Rayo, Quebrada Biscoyol, Río Ostional, Quebrada Seca, Río Montaña, Río Nosara, Quebrada Peladas, Río Rempujo, Quebrada Corea, Estero García y Quebrada Guiones.

En la superficie, el rango salino se ubicó entre 33.2 y 33.8 PSU, valores típicos del Agua Superficial Tropical (AST). Esta masa de agua se localiza en el centro del Pacífico Tropical a lo largo de los $10^{\circ} \mathrm{N}$ de latitud. Se caracteriza por presentar temperaturas superiores a $\operatorname{los} 25^{\circ} \mathrm{C}$ y salinidades bajas ( $<34 \mathrm{PSU}$ ), producto de un exceso anual de la precipitación $(\mathrm{P})$ sobre la
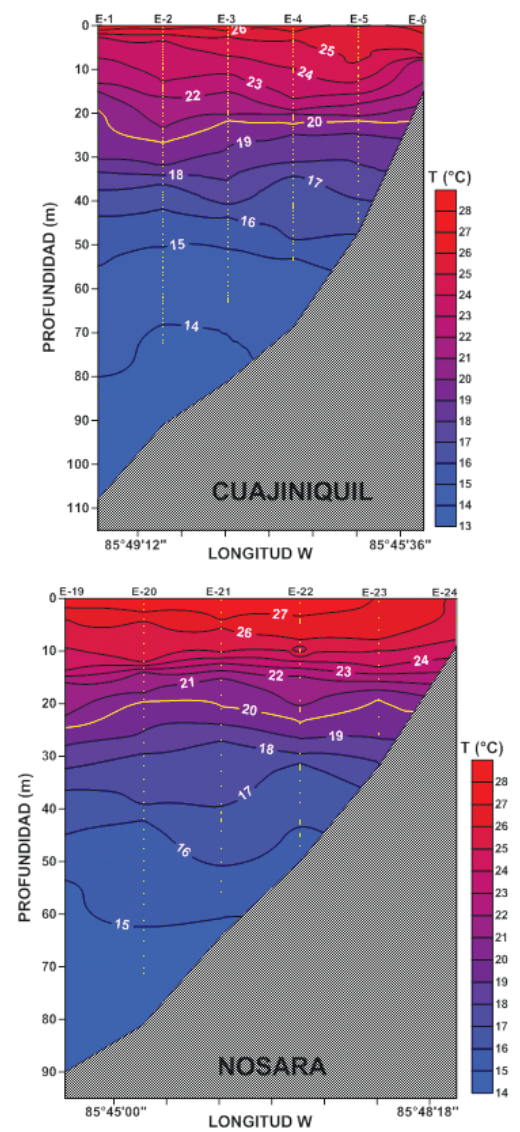

evaporación (E) del orden de los $500 \mathrm{~mm}$ (Brenes, 1984). A $20 \mathrm{~m}$ de profundidad, el campo salino se mostró mucho más homogéneo. Un núcleo de baja salinidad superficial frente a Ostional (Fig. 3D), se extiende hasta el límite sur de la región estudiada. Este comportamiento podría asociarse a un mayor aporte de agua dulce proveniente de los ríos ubicados en la parte sur. Aproximadamente a $3 \mathrm{~km}$ al sur de Ostional, se localiza la desembocadura del río Nosara, la mayor de la Península de Nicoya, y según Alvarado (1985) su corriente fluye al frente de la playa de Ostional con dirección norte, el cual, junto con los ríos Ostional y Montaña, son los que depositan la mayor cantidad de agua dulce en época de invierno.
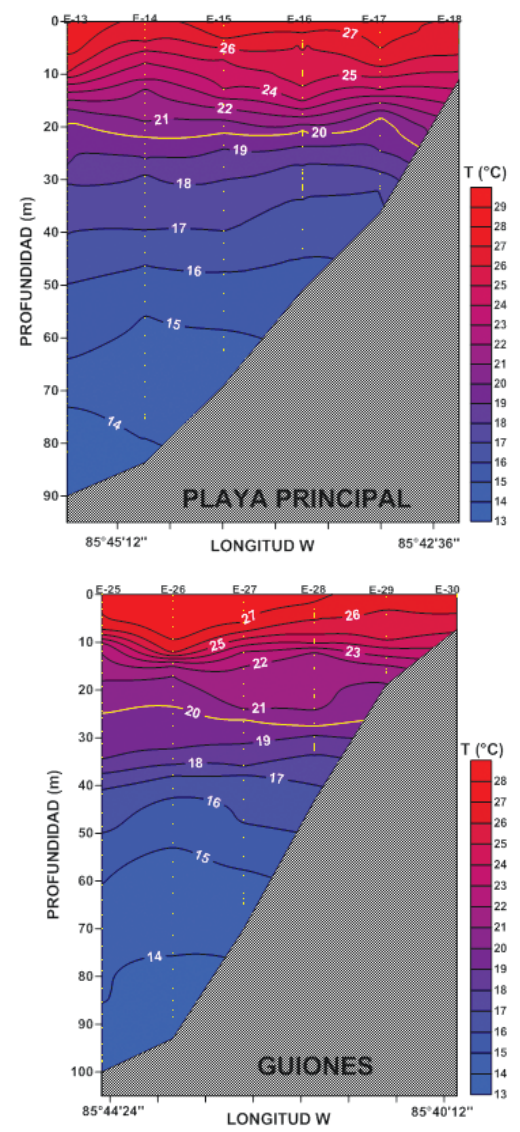

Fig. 4. Distribuciones verticales de temperatura $\left({ }^{\circ} \mathrm{C}\right)$ en cuatro transectos

Fig. 4. Vertical distribution of temperature $\left({ }^{\circ} \mathrm{C}\right)$ in four transects 
El gradiente vertical de temperatura en los primeros $20 \mathrm{~m}$ de profundidad fue de $0.3^{\circ} \mathrm{C} . \mathrm{m}^{-1}$, en tanto que el salino apenas alcanzó 0.05 PSU.m ${ }^{-1}$.

Para analizar la estructura vertical de los campos termohalinos, se escogieron cuatro transectos ubicados frente a Cuajiniquil, Playa Principal, Nosara y Guiones. Los resultados obtenidos se encuentran en las Figs. 4 y 5.

En todos los transectos se localiza una capa de mezcla isotérmica, cuya profundidad apenas alcanza los $10 \mathrm{~m}$. Es un estrato muy homogéneo, con temperaturas de $27^{\circ} \mathrm{C}$, en el cual la energía necesaria para generar los procesos de mezcla se deriva, en buena parte, del campo de viento superficial.
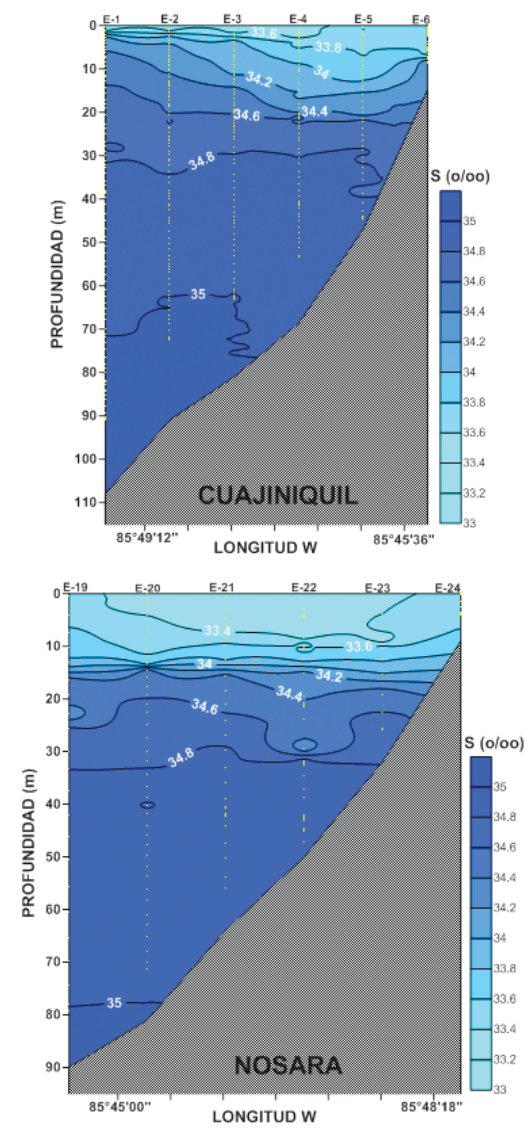

La posición de la termoclina, definida por la profundidad de la isoterma de $20^{\circ} \mathrm{C}$, se ubica a los $20 \mathrm{~m}$ desde Ostional hasta Cuajiniquil; sin embargo, en el extremo sur de la región, desde Ostional hasta Guiones, se puede observar ligeramente más profunda, alrededor de los $25 \mathrm{~m}$. En el transecto frente a Ostional (Fig. 4, Playa Principal), la inclinación ascendente de las isotermas, desde los 60 $\mathrm{m}$ de profundidad en la estación 14 , se asocia a un giro ciclónico de un diámetro cercano a los $3 \mathrm{~km}$, en el cual las aguas más densas y frías se localizan en su centro. Un giro anticiclónico se localiza inmediatamente después, centrado en la estación 16, con un diámetro de $4 \mathrm{~km}$. Estas estructuras subsuperficiales están en concordancia con lo observado a
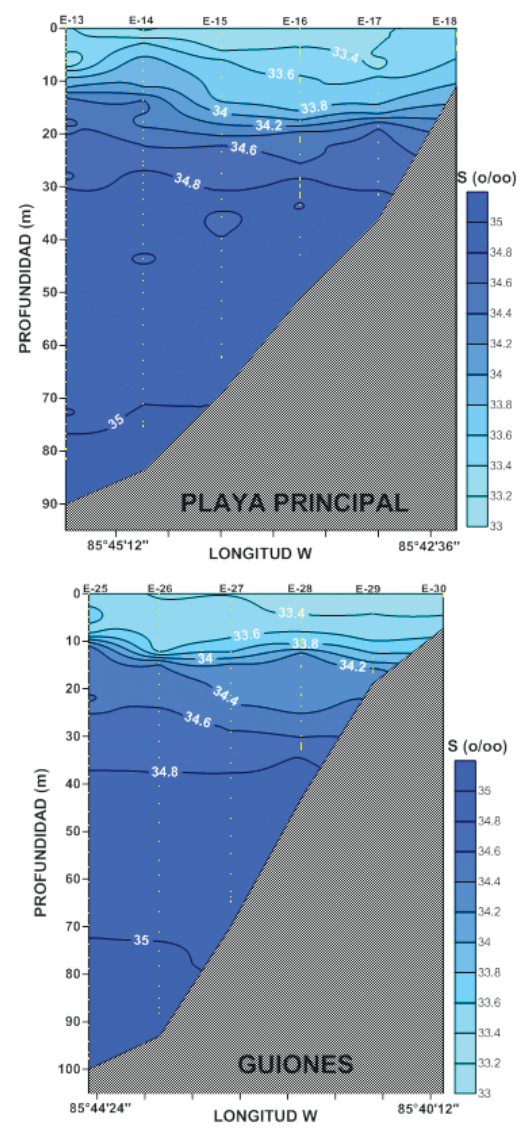

Fig. 5. Distribuciones verticales de salinidad (psu) en cuatro transectos

Fig. 5. Vertical distribution of salinity (psu) in four transects 
nivel superficial en la Fig. 3A. Swimmer et al. (2006) encontraron, al analizar la captura incidental de tortugas lora (Lepidochelys olivacea) en el Pacífico Tropical Oriental, que más del $95 \%$ del tiempo estas tortugas se mueven en aguas sobre los $60 \mathrm{~m}$ de profundidad con temperaturas entre los 22 y $28^{\circ} \mathrm{C}$.

La salinidad en los estratos más superficiales de la columna es menor en los transectos ubicados al sur de la región (Nosara y Guiones) (Fig. 5). La isohalina de 33.4 PSU se extiende hasta los $10 \mathrm{~m}$ de profundidad en Nosara, contrastando con lo observado en Cuajiniquil, donde la salinidad más baja fue de 33.6 PSU.

Entre los 30 y $80 \mathrm{~m}$ de profundidad, se localiza el núcleo de alta salinidad, asociado al Agua Subsuperficial Subtropical (ASS). Esta masa de agua, caracterizada por el máximo de salinidad cercano a las 35 PSU en el diagrama T-S (Fig. 6), ocupa los niveles subtermoclinos. El origen de esta masa de agua se sitúa en el Océano Pacífico Sur y se desplaza atravesando el ecuador hacia el Hemisferio Norte. Cuando alcanza el ecuador sufre la influencia de la
Subcorriente Ecuatorial, pasando por considerables procesos de mezcla, resultando así una masa de agua con temperaturas y salinidades menores de $16^{\circ} \mathrm{C}$ y $35 \mathrm{PSU}$, respectivamente (Brenes, 1984).

Para el análisis de la variación latitudinal (sur-norte), se definió un transecto formado por las estaciones 27, 21, 16, 9 y 3 . Las isotermas en los niveles sobre la termoclina muestran una inclinación ascendente en dirección norte (Fig. 7A). Este mismo comportamiento se observa en el campo vertical salino (Fig. 7B). En el extremo norte (Cuajiniquil), el tope de la termoclina (isoterma de $25^{\circ} \mathrm{C}$ ) se localiza a menos de $5 \mathrm{~m}$ de la superficie del mar (Fig. 7A). Una haloclina bien definida y centrada en los 20 $\mathrm{m}$ de profundidad puede observarse en la figura 7B, en la misma posición donde se encuentra la termoclina. Aguas poco salinas se extienden desde la parte sur de la región (Guiones) hasta San Juanillo (estación 9). Al norte de la estación 21 frente a Nosara, se observa un levantamiento hacia la superficie de las isohalinas. Estos resultados muestran una asimetría hidrográfica entre las regiones

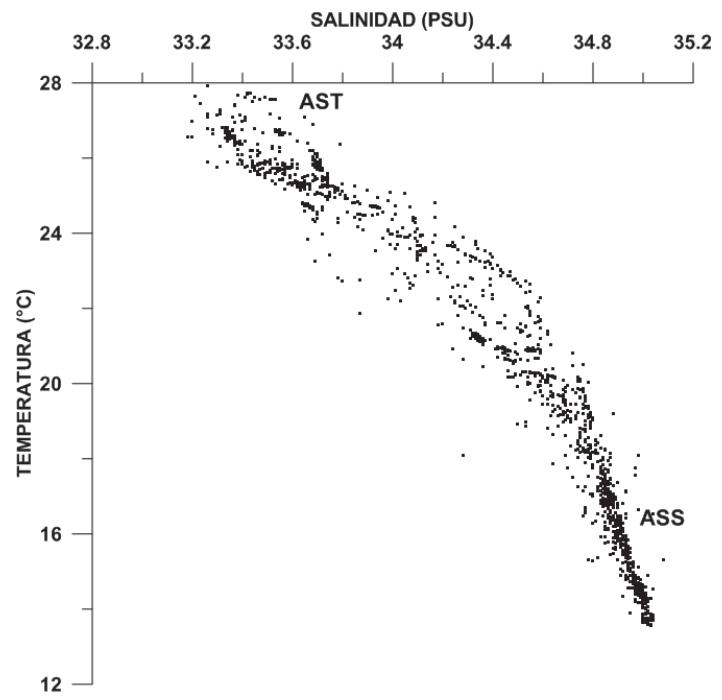

Fig. 6. Diagrama T-S dispersivo. AST: Agua Superficial Tropical, ASS: Agua Subsuperficial Subtropical Fig. 6. T-S dispersive diagram. STW: Surface Tropical Water. SSW: Subsurface Subtropical Water 

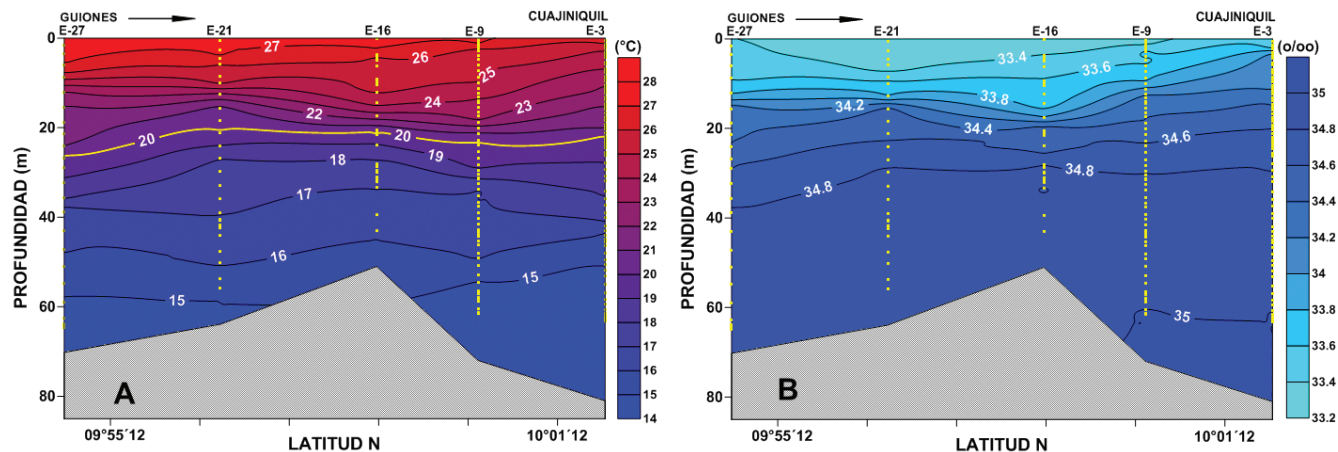

Fig. 7. Distribuciones verticales de temperatura $\left({ }^{\circ} \mathrm{C}\right)(\mathrm{A})$ y salinidad (PSU) (B) en un transecto latitudinal a lo largo de la región estudiada

Fig. 7. Vertical distribution of temperature $\left({ }^{\circ} \mathrm{C}\right)(\mathrm{A})$ and salinity (PSU) (B) in a latitudinal transect across the study region

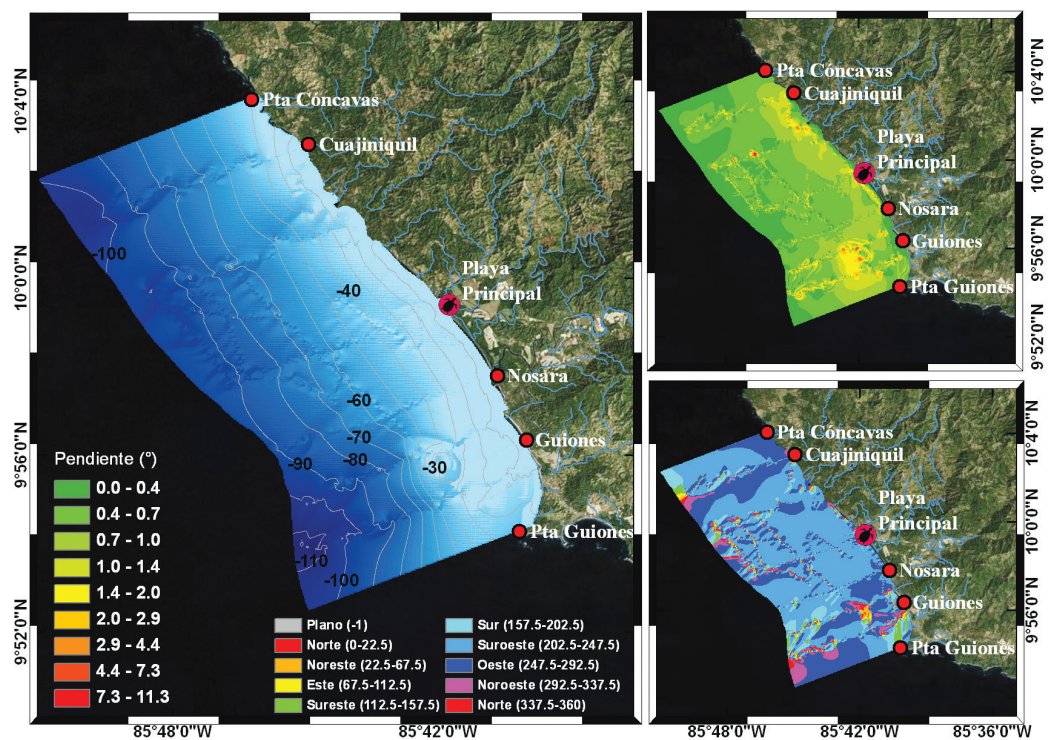

Fig. 8. A) Contornos batimétricos, B) rugosidad representada por el grado de inclinación de las pendientes y $\mathrm{C}$ ) el aspecto del terreno

Fig. 8. A) Bathymetric, B) roughness represented by the degree of inclination of the slopes and C) appearance of the field

sur y norte del área estudiada, la cual podría tener alguna influencia en las arribadas de las tortugas, ya que entre la desembocadura del río Nosara y Punta India anidan masivamente las tortugas lora, mientras que es menor en el extremo sur del Parque Nacional, entre Punta Guiones y Nosara.

En relación con la batimetría de la región, las isóbatas se muestran paralelas a la costa, y sobresale una elevación $(\sim 20 \mathrm{~m})$ en el extremo sur de la zona entre Punta Guiones y Ostional (Figs. 8A y 9). Entre las isóbatas de 10 y $20 \mathrm{~m}$ frente a San Juanillo y en las cercanías de Punta Guiones, donde se localizó la elevación antes mencionada, se obtuvieron las pendientes con mayor grado de inclinación (Figs. 8B y 9). 
La pendiente no excede los $14.5^{\circ}$ y el patrón general es una inclinación leve con dirección suroeste, es decir, desde la costa hacia el mar abierto, no obstante, algunas zonas registran una inclinación en dirección oeste (Fig. 8C). Los extremos norte y sur del área son los que presentan mayores irregularidades batimétricas, mientras que la zona centrada en Ostional muestra un relieve mucho más homogéneo y suave. Según Márquez (2000), existen ciertas características en las playas que definen cuál o cuáles son las especies más probables que aniden en ellas: a) playas abiertas, continentales, aisladas, con poca pendiente (cerca de $5^{\circ}$ ), de mediana energía y generalmente limitadas en su parte terrestre por esteros o marismas, son las más visitadas por tortugas del género Lepidochelys; b) playas abiertas o bahías, continentales o insulares, con mediana o poca pendiente (entre $5^{\circ}$ y $10^{\circ}$ ), de mediana o baja energía, arbustiva en su porción terrestre y franqueada su zona marítima por barreras coralinas o rocosas, a poca profundidad, son las más comunes para las tortugas de los géneros Dermochelys, Caretta y Chelonia; y c) playas abiertas, generalmente continentales, de alta energía, pendiente pronunciada (más de $10^{\circ}$ ) y libres de barreras en su porción marítima, son las más visitadas por Dermochelys.

La figura 9 es una representación tridimensional de la batimetría de la zona. Las áreas más irregulares se localizan al sur de la comunidad de Guiones. El $\Delta \mathrm{Z} / \Delta \mathrm{X}$ (el gradiente horizontal de la profundidad) es más pronunciado al sur de Ostional con un valor que ronda los $10 \mathrm{~m} /$ $\mathrm{km}$, casi el doble del valor que se tiene al norte de la región frente a San Juanillo o Punta Cóncavas.

\section{CONCLUSIONES}

Durante este estudio, las temperaturas superficiales se ubicaron entre los 25.5 y $27.5^{\circ} \mathrm{C}$, y el rango salino superficial estuvo entre 33.2 y 33.8 PSU. La termoclina se localizó a los $20 \mathrm{~m}$ en el extremo norte de la región, mientras que en el extremo sur, desde Ostional hasta Guiones, se

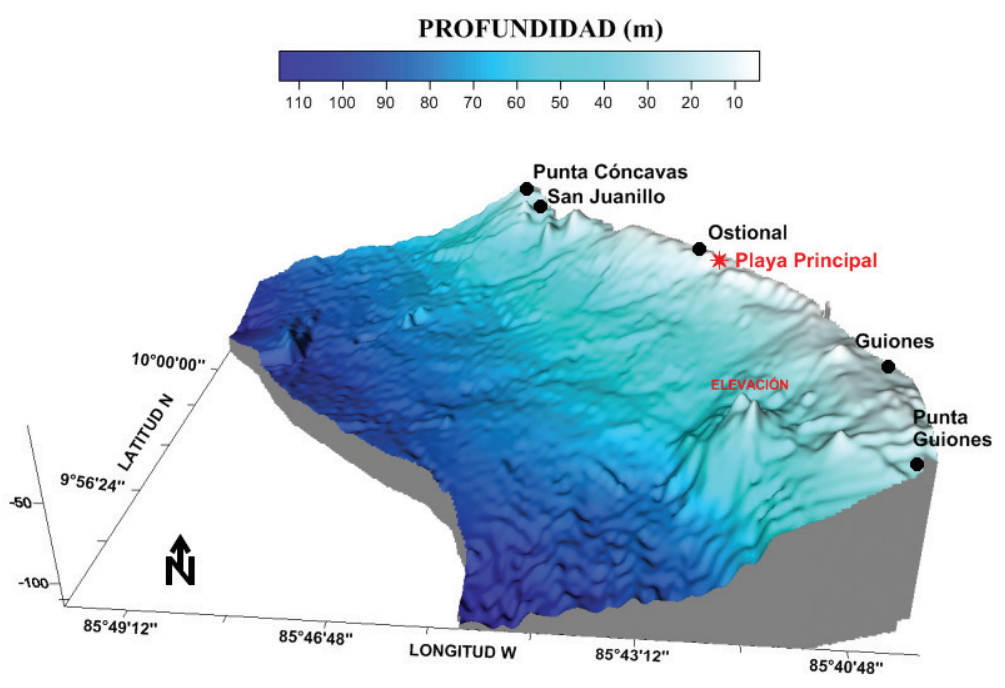

Fig. 9. Mapa batimétrico tridimensional

Fig. 9. Three-dimensional bathymetric map 
observó ligeramente más profunda, alrededor de los $25 \mathrm{~m}$. El espesor de la capa de mezcla fue de $10 \mathrm{~m}$ en toda el área estudiada. Dos giros, uno ciclónico y otro anticiclónico, se registraron en la región frente a Ostional. Los estratos superficiales estuvieron ocupados por el Agua Superficial Tropical (AST), con salinidades inferiores a 34 PSU y temperaturas superiores a $26^{\circ} \mathrm{C}$, y los niveles intermedios, por el Agua Subsuperficial Subtropical (ASS), caracterizada por un núcleo salino cercano a las 35 PSU. La batimetría de la región muestra isóbatas paralelas a la costa, con una elevación alrededor de los 20 $m$ de profundidad en el extremo sur de la zona estudiada. El grado de inclinación o pendientes no sobrepasaron los $14.5^{\circ}$.

\section{AGRADECIMIENTOS}

Este trabajo se llevó a cabo gracias al apoyo económico brindado por la Universidad Nacional y la International Students Volunteers (ISV-CR). Nuestro agradecimiento al Área de Conservación Tempisque (ACT-MINAET), especialmente al Refugio Nacional de Vida Silvestre Ostional y a la Asociación de Pescadores de San Juanillo, quienes colaboraron en la toma de datos.

\section{BIBLIOGRAFÍA}

Alvarado, M. A. (1985). Tasa de éxito de eclosión de nidos naturales de la tortuga marina Lepidochelys olivacea (Eschscholtz, 1829), en el Refugio Nacional de Fauna Silvestre Ostional, Guanacaste, Costa Rica. Tesis de Licenciatura no publicada. Universidad de Costa Rica.

Amador, J. A., Alfaro, E. J., Lizano, O. G. \& Magaña, V. O. (2006). Atmospheric forcing of the eastern tropical Pacific: A review. Progr. Oceanogr., 69, 101-142.

Bergoing, J. P. \& Brenes, L. G. (1977). Mapa geomorfológico de Costa Rica. Según imágenes tomadas por el satélite Landsat. San José, Costa Rica: Instituto Geográfico Nacional.

Brenes, C. L. (1984). Las masas de agua del Océano Pacífico Tropical frente a Costa Rica. UNICIENCIA, 1(1), 31-36.

Brenes, C. L., Lavín, M. F. \& Mascarenhas, A. S., Jr. (2008). Geostrophic circulation between the Costa Rica Dome and Central America. Deep-Sea Res., (I), 55(5), 608-629.

Cornelius, S. E. \& Robinson, D. C. (1987). Post-nesting movements of female olive ridley turtles tagged in Costa Rica. Vida Silvestre Neotrop., 1(1), 12-23.

Chelton, D. B., Freilich, M. H. \& Esbensen, S. K. (2000a). Satellite observations of the wind jets off the Pacific coast of Central America. Part I: case studies and statistical characteristics. Mon. Wea. Rev., 128(7), 1993-2018.

Chelton, D. B., Freilich, M. H. \& Esbensen, S. K. (2000b). Satellite observations of the wind jets off the Pacific coast of Central America. Part II: regional relationships and dynamical considerations. Mon. Wea. Rev., 128, 2019-2043.

Fiedler, P. C. \& Talley, L. D. (2006). Hydrography of the eastern Pacific: A review. Progr. Oceanogr., 69, 143-180.

Kessler, W. S. (2006). The circulation of the eastern tropical Pacific: A review. Progr. Oceanogr., 69, 181-217.

Lizano, O. G. (2007). Climatología del viento y oleaje frente a las costas de Costa Rica. Cienc. Tecn., 25(1-2), 43-56.

Márquez, R. (2000). Las tortugas marinas y nuestro tiempo. México: Fondo de Cultura Económica. 
Plotkin, P. (2010). Nomadic behaviour of the highly migratory olive ridley sea turtle Lepidochelys olivacea in the eastern tropical Pacific Ocean. Endang. Species Res., 13, 33-40.

Polovina, J. J., Howell, E., Kobayashi, D., Seki, M. P. (2001). The transition zone chlorophyll front, a dynamic global feature defining migration and forage habitat for marine resources. Prog. Oceanogr., 49, 469-483.

Polovina, J. J., Balazs, G. H., Howell, E. A., Parker, D. M., Seki, M. P. \& Dutton, P. H. (2004). Forage and migration habitat of loggerhead (Caretta caretta) and olive Ridley (Lepidochelys olivacea) sea turtles in the central North Pacific Ocean. Fish. Oceanogr., 13, 36-51.

SURFER 10. (2011). Surfer ${ }^{\circledR}$ Contouring and 3D Surface Mapping for Scientists and Engineers, version 10
www.GoldenSoftware.com. Colorado, EE.UU.: Golden Software, Inc.

Swimmer, Y., Arauz, R., McCracken, M., Ballestero, J., Musyl, M., Bigelow, K. \& Brill, R. (2006). Diving behavior and delayed mortality of olive ridley sea turtles Lepidochelys olivacea after their release from longline fishing gear. Mar. Ecol. Prog. Ser., 323, 253-261.

Swimmer, Y., McNaughton, L., Foley, D., Moxey, L. \& Nielsen, A. (2009). Movements of olive ridley sea turtles Lepidochelys olivacea and associated oceanographic features as determined by improved light-based geolocation. Endang. Species Res., 10, 245-254.

Tosi, J. A., Jr. (1969). Mapa Ecológico. San José, Costa Rica: Centro Científico Tropical.

Valverde, R. (2007). Global Assessment of Arribada Olive ridley Sea Turtles. Louisiana, EE.UU.: USWS. 